\title{
Breather statics and dynamics in Klein-Gordon chains with a bend
}

\author{
J. Cuevas* \\ Grupo de Física No Lineal, Departamento de Física Aplicada I, ETSI Informática, Universidad de Sevilla, \\ Avenida Reina Mercedes, s/n, 41012-Sevilla, Spain \\ P. G. Kevrekidis ${ }^{\dagger}$ \\ Department of Mathematics and Statistics, University of Massachusetts, Amherst, Massachusetts 01003-4515, USA
}

(Received 30 June 2003; published 20 May 2004)

\begin{abstract}
In this paper, we examine a nonlinear model with an impurity emulating a bend. We justify the geometric interpretation of the model and connect it with earlier work on models including geometric effects. We focus on both the bifurcation and stability analysis of the modes that emerge as a function of the strength of the bend angle, but we also examine dynamical effects including the scattering of mobile localized modes (discrete breathers) off of such a geometric structure. The potential outcomes of such numerical experiments (including transmission, trapping within the bend as well as reflection) are highlighted and qualitatively explained. Such models are of interest both theoretically in understanding the interplay of breathers with curvature, but also practically in simple models of photonic crystals or of bent chains of DNA.
\end{abstract}

DOI: 10.1103/PhysRevE.69.056609

PACS number(s): 05.45.Yv, 63.20.Pw, 63.20.Ry, 63.50.+x

\section{INTRODUCTION}

In the last decade, intrinsic localized modes (ILMs), or discrete breathers as they are also termed, have become a topic of intense theoretical and experimental investigation; see, e.g., Ref. [1] for a number of recent reviews on the topic. Per their inherent ability to bottleneck and potentially transport the energy in a coherent fashion, such exponentially localized in space and periodic in time entities have come to be of interest in a variety of contexts. These range from nonlinear optics and arrays of waveguides [2] to BoseEinstein condensates inside optical lattice potentials [3] and from prototypical models of nonlinear springs [4] to Josephson junctions [5] and dynamical models of the DNA double strand [6].

One of the playgrounds that have most recently been added to this long list of ILM applications consists of nonlinear photonic crystal waveguides and circuits [7]. In connection to this context, an issue that becomes very relevant (see, e.g., the models developed in Ref. [7]) is the interplay of nonlinearity and geometry as, typically in photonic crystal waveguide arrays, two-dimensional or quasi-onedimensional settings with bends $[7,8]$ become relevant.

We should note here that the interplay of nonlinearity and geometry has been increasingly appreciated in the ILM literature. From the long range interactions on a fixed curved substrate [9] to lattice-substrate feedback models [10] and from lattice junctions with different masses [11] to semicircular, polymerlike chains [12] and geometrically motivated, bent models of DNA [13-16], the geometry can significantly affect the static (inducing, e.g., multistability) and dynamic (causing, e.g., a variety of outcomes in the ILM interaction with curvature) properties of the relevant lattice model.

\footnotetext{
*Electronic address: jcuevas@us.es

†Electronic address: kevrekid@math.umass.edu
}

In the present work, motivated by these studies we examine the nonlinear Klein-Gordon variant of a model introduced recently in the context of the discrete nonlinear Schrödinger equation (DNLS) in Ref. [8]. This is a prototypical model emulating the geometry of a lattice bend, by the inclusion in the vicinity of the bend of next-nearest neighbor interaction due to the proximity of these neighbors in this context (see, e.g., Fig. 1).

We will examine this bend in the framework of a soft and a hard Klein-Gordon interaction potential, namely the Morse and the hard $\phi^{4}$ potentials, respectively. Apart from the inherent interest of the interaction, at the static as well as dynamical level, of discrete breathers with the "geometric impurity" induced by the bend, the model may be relevant to a number of applications. In particular, the DNLS serves as the envelope wave equation (at the discrete level) for Maxwell's equation, hence, for the photonic applications, it may be of more interest to identify the properties of the corresponding

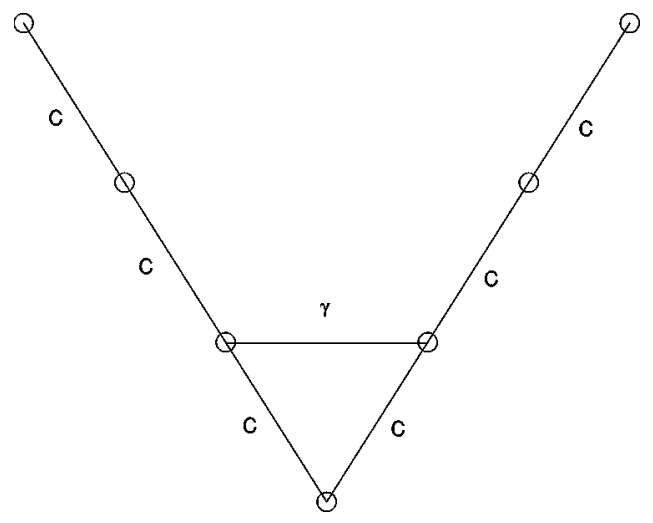

FIG. 1. Schematic presentation of a bend in connection with the discrete equation (2). The parameter $\gamma$ stands for the bend-induced interaction between the next-nearest neighbors (in the vicinity of the bend), whereas $C$ represents the nearest neighbor interaction in the rectilinear chain. 


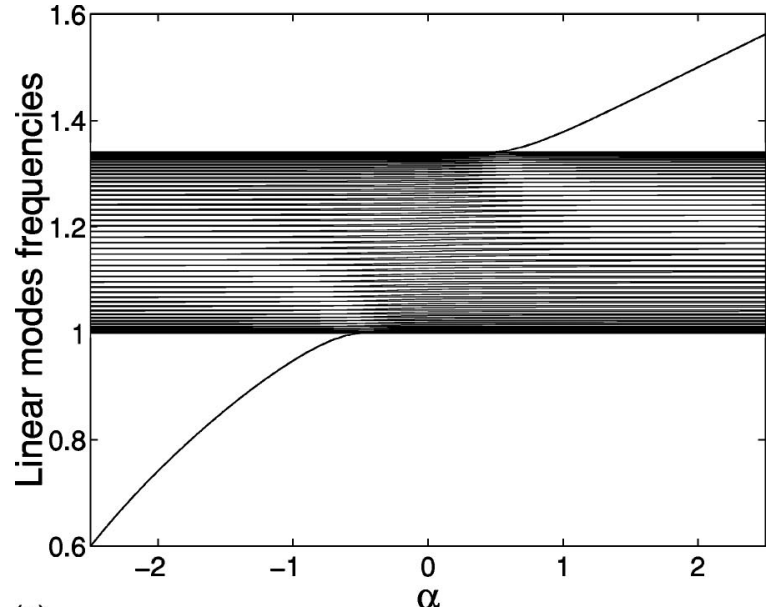

(a)

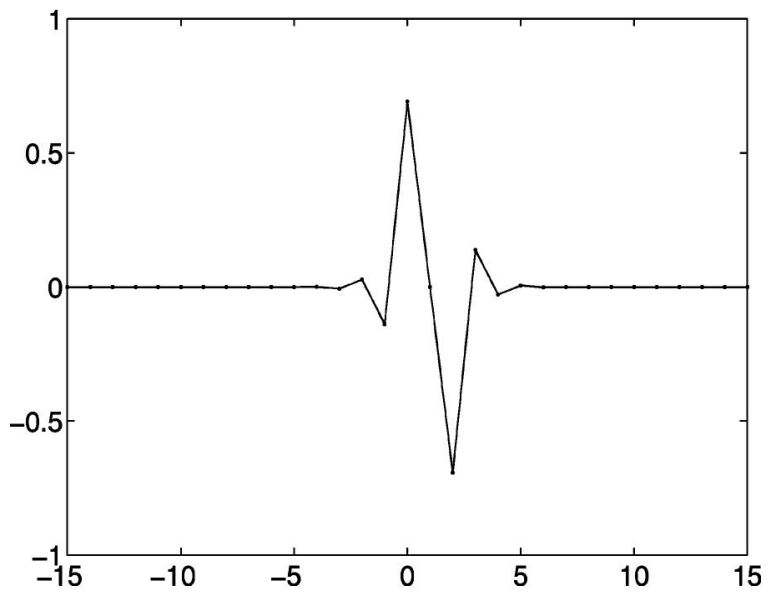

(b)

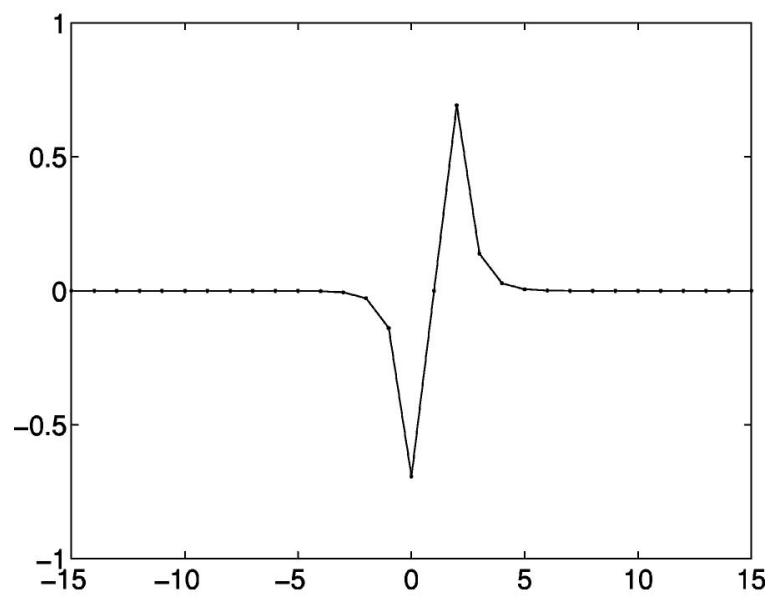

(c)

FIG. 2. (a) Linear mode spectrum with respect to the bending parameter $\alpha=\gamma / C$ for a coupling constant $C=0.20$. (b,c) Profile of the impurity mode for $\alpha>0$ (b) or $\alpha<0$ (c) and $|\alpha|=2.5$. Note that the localization of the impurity modes is non-negligible only for high values of $\alpha$.

Klein-Gordon model. Furthermore, this may also serve as a simple dynamical model for understanding the interaction of a denaturation bubble in DNA [6,17] with the local helical geometry of the double strand. This is similar in spirit to the
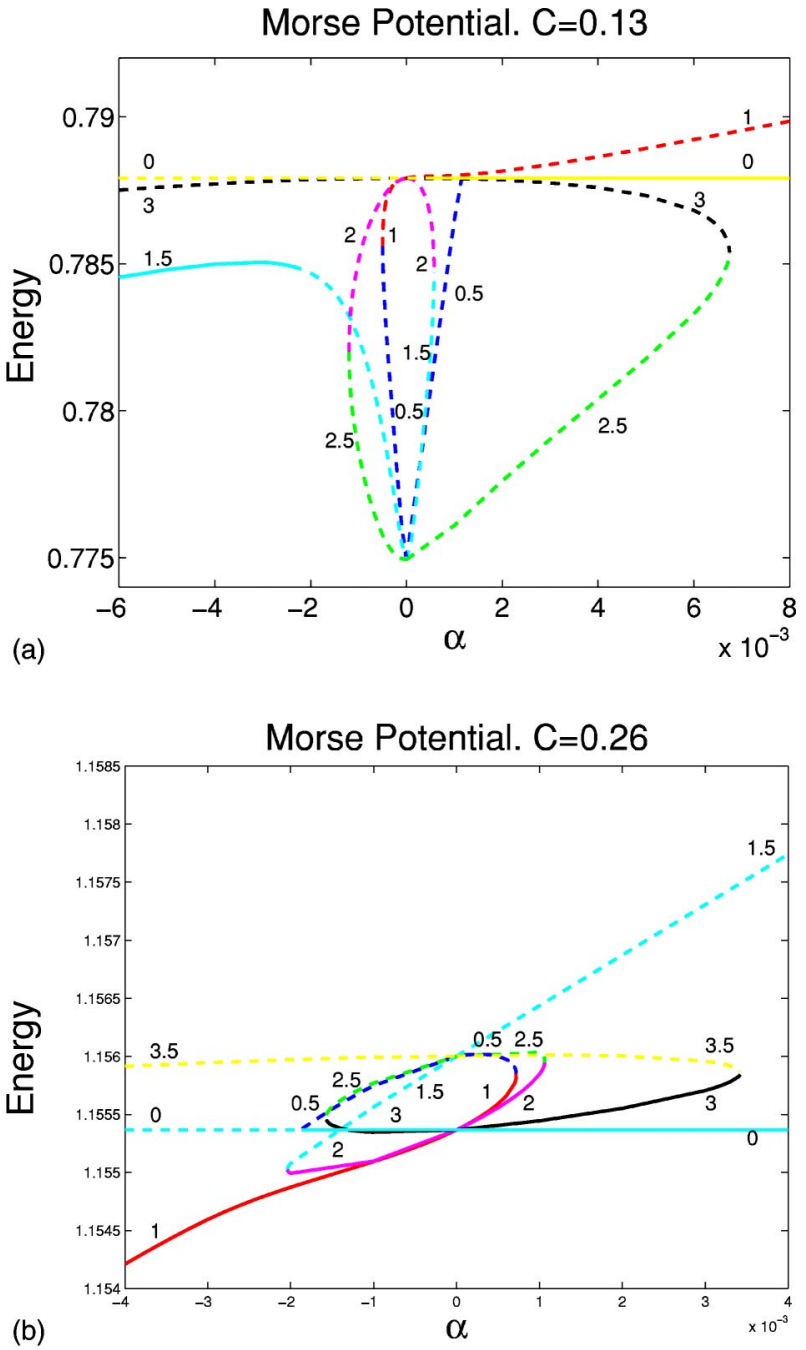

FIG. 3. Bifurcation diagram for the solutions with a Morse onsite potential. The energy of the breathers is plotted as a function of the bending parameter $\alpha=\gamma / C$. The coupling constant is $C=0.13$ (top) or $C=0.26$ (bottom). The numbers indicate the site where the solutions are centered; an integer number corresponds to a sitecentered solution, whereas a half-integer number corresponds to a bond-centered solution. Stable solutions (for an infinite system) are represented by full lines whereas unstable solutions are represented by dashed lines.

earlier study of Ref. [19], where the helicoidal geometry was argued to induce a non-nearest neighbor interaction across the double strand. Notice, also, that we follow a slightly different path than Ref. [8], by examining not only static but also dynamic properties of the model and, in particular, the potential outcomes of the breather-bend interaction.

In the following section, we will present the model equation of interest, while in Sec. III, we will study its linear modes. In Sec. IV, we will examine the static properties of the two models, by examining the bifurcations of breathing modes as a function of the "bend parameter" $\gamma$. In Sec. V, the corresponding dynamics properties of the breather-bend interaction will be presented. Finally, in Sec. VI, we summarize our findings and present our conclusions. 


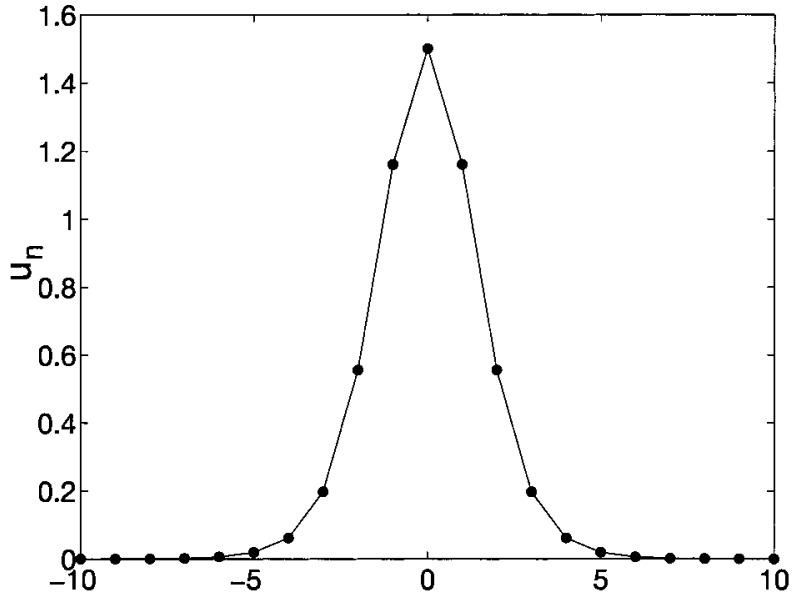

(a)

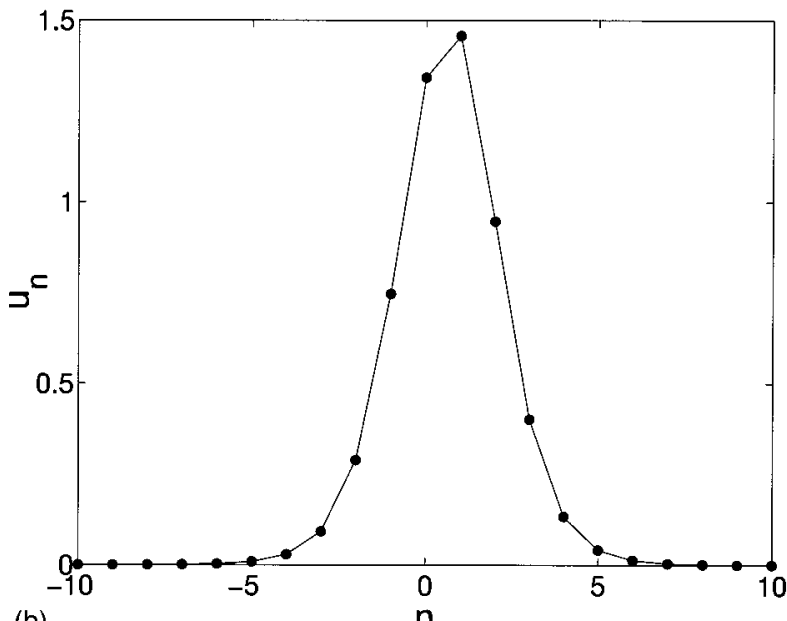

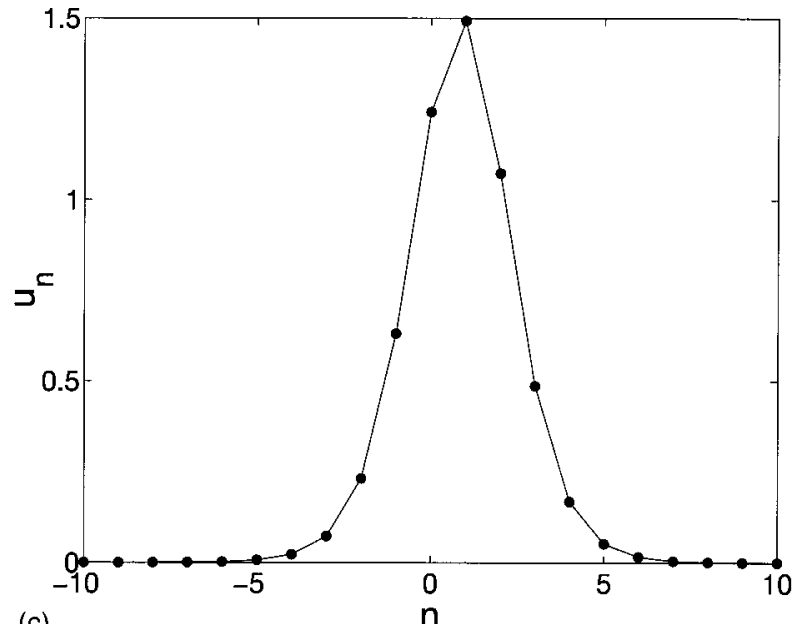

(c)

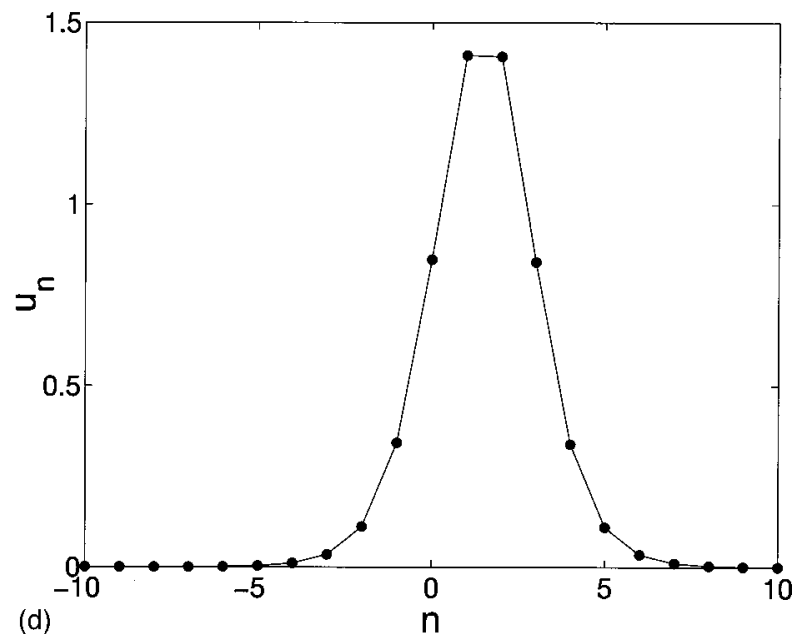

FIG. 4. Spatial profiles of the breathers centered at $n=0$ (a), $n=0.5$ (b), $n=1$ (c), and $n=1.5$ (d) for $\alpha=0.0005$. The results correspond to a chain of oscillators with an on-site Morse potential with parameters $\omega_{\mathrm{b}}=0.8$ and $C=0.26$.

\section{THE MODEL}

In accordance with the above description, the Hamiltonian of the Klein-Gordon chains of interest will be given by

$H=\sum_{n}\left(\frac{1}{2} \dot{u}_{n}+V\left(u_{n}\right)+\frac{C}{2}\left(u_{n+1}-u_{n}\right)^{2}+\frac{\gamma}{2}\left(u_{n+1}-u_{n-1}\right)^{2} \delta_{n, 0}\right)$,

thus the bending point is located at the particle $n=0$ and implies, as is graphically indicated in Fig. 1, a geometrically induced coupling of next-nearest neighbors (NNN) adjacent to the bend site.

We should clarify here the nature of the configuration and of the dynamics. The displacements $u_{n}$ represent an "external" field such as, e.g., the electric field in an array of optical waveguides or the stretching of a base pair in a DNA chain [6]. The chain of Fig. 1 should be considered as being geometrically fixed on the plane of the paper. The dynamics of the displacements is transverse, i.e., perpendicular to the plane of the bent chain. In such a setting the coefficient of the interaction between adjacent sites is determined by the (fixed) geometric proximity of the nodes constituting the chain. For a derivation of the discrete model in the first setting and an explanation of the dependence of the interaction coefficient on the geometry of the configuration and the internodal distance, see, e.g., Ref. [18]. In this context, it is natural to assume that the effect of the bend (due to the geometric proximity of sites $n=1$ and $n=-1$ ) will be to induce the NNN coupling between them (cf. Ref. [18]).

$V(u)$ is the on-site potential which is chosen to be of the (soft) Morse type, $V(u)=[\exp (-u)-1]^{2} / 2$, or of the (hard) $\phi^{4}$ type, $V(u)=x^{2} / 2+x^{4} / 4 ; C$ is a coupling constant between nearest neighbors (NN) whose interaction will be harmonic (i.e., connected by linear springs) for the purposes of the present work.

From the Hamiltonian (1), the following dynamical equations can be obtained:

$$
\begin{aligned}
\ddot{u}_{n}+ & V^{\prime}\left(u_{n}\right)+C\left(u_{n+1}+u_{n-1}-2 u_{n}\right)+\gamma\left[\left(u_{n}-u_{n-2}\right) \delta_{n, 1}\right. \\
& \left.+\left(u_{n}-u_{n+2}\right) \delta_{n,-1}\right]=0 .
\end{aligned}
$$

A natural bifurcation/continuation parameter that we will use for the purposes of our study is $\alpha \equiv \gamma / C$. The relevant ratio is a natural measure of the relative strength of the different neighbor interactions (the bend-based NNN one and the uni- 

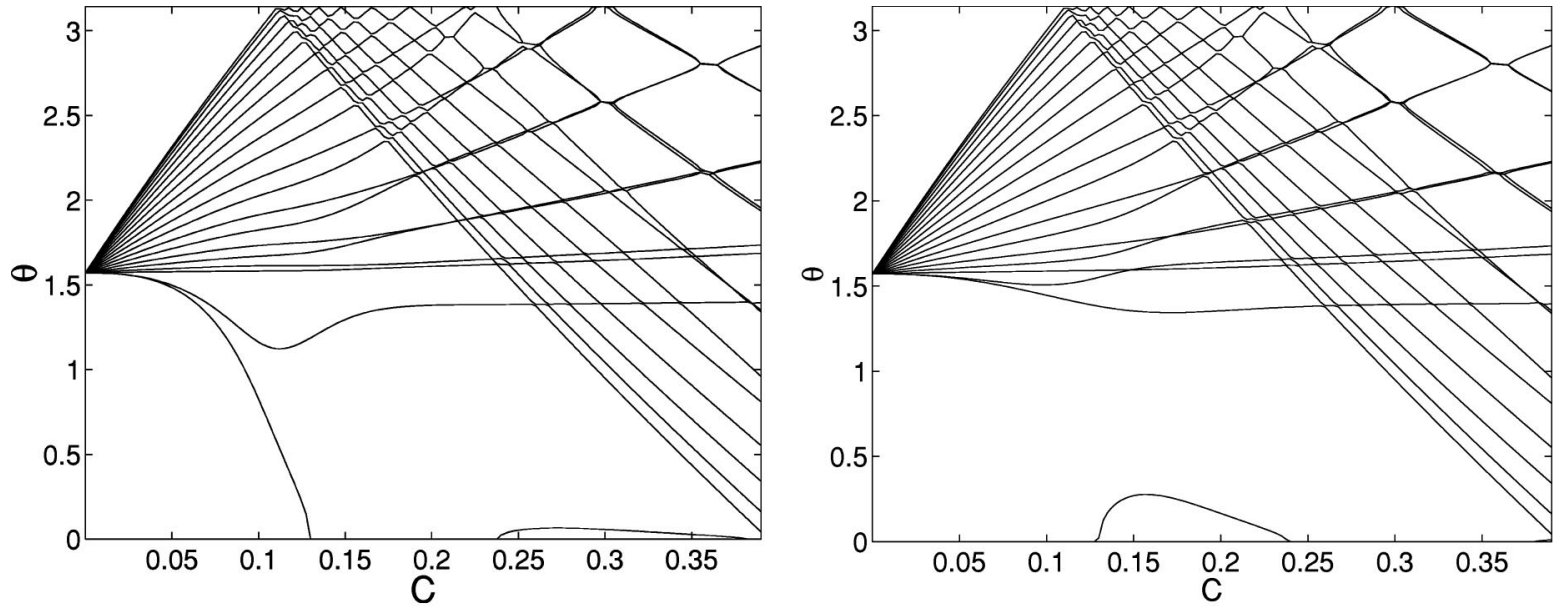

(a)

(c)
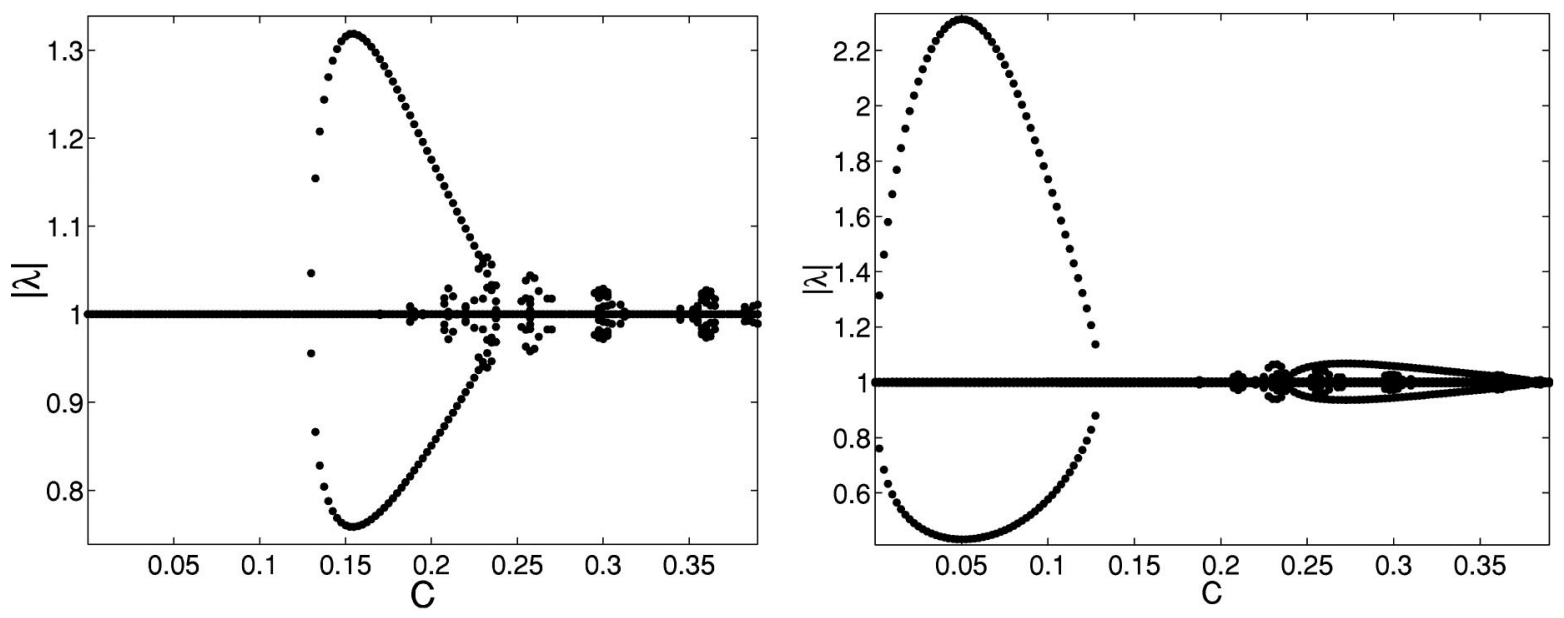

(d)

FIG. 5. Evolution with respect to the coupling constant of the Floquet multiplier arguments $\theta$ (left) and the modulus of the Floquet multipliers (right) for a one-site (top) and a two-site (bottom) breather with Morse on-site potential with frequency $\omega_{\mathrm{b}}=0.8$. The one-site breather is stable for $C \in(0,0.1297)$ and for $C \in(0.2382,0.4)$ it recovers its stability except for size-dependent instability bubbles via oscillatory and subharmonic bifurcations. The 2 -site breather is stable for $C \in(0.1300,0.2381)$.

formly distributed $\mathrm{NN}$ one). This ratio can also be interpreted geometrically (in the spirit of Fig. 1). In particular, it can be related to the wedge angle $\phi$ through the relation $\alpha$ $=1 /(2[1-\cos (\phi)])$. For the NNN approximation to be realistic, $\phi$ must be larger than $60^{\circ}$, hence, equivalently, $\alpha<1$. Notice that while the geometric interpretation of the moment prompts us to typically examine the regime of $\alpha \in(0,1]$, it is of inherent mathematical interest to examine broader parameter ranges, and therefore some of our results below will be presented for values of $\alpha$ outside this range.

\section{LINEAR MODES}

Some of the properties of discrete breathers are related to the existence or nonexistence of linear localized modes. These modes appear as the bend is introduced by modifying the coupling, which is effectively equivalent to the introduction of an inhomogeneity in the curvature at the bottom of the intersite potential [20].
The linear modes can be obtained from the linearized form (around the uniform state $u_{n}=0$ ) of the dynamical equations (2):

$$
\begin{aligned}
\ddot{u}_{n}+ & \omega_{o}^{2} u_{n}+C\left(2 u_{n}-u_{n-1}-u_{n+1}\right)+\gamma\left[\left(u_{n}-u_{n-2}\right) \delta_{n, 1}\right. \\
& \left.+\left(u_{n}-u_{n+2}\right) \delta_{n,-1}\right]=0
\end{aligned}
$$

with $\omega_{o}^{2}=1$. The linear modes can be calculated using the lattice Green's function [21,22]. The frequency of the localized linear modes (also referred to as impurity modes) is thus given by

$$
\omega_{\text {imp }}^{2}=\omega_{o}^{2}+\frac{C(2 \alpha+1)^{2}}{2 \alpha}=\omega_{o}^{2}+\frac{(2 \gamma+C)^{2}}{2 \gamma},
$$

and the inhomogeneity parameter is related to the frequency of the impurity modes through the relation 


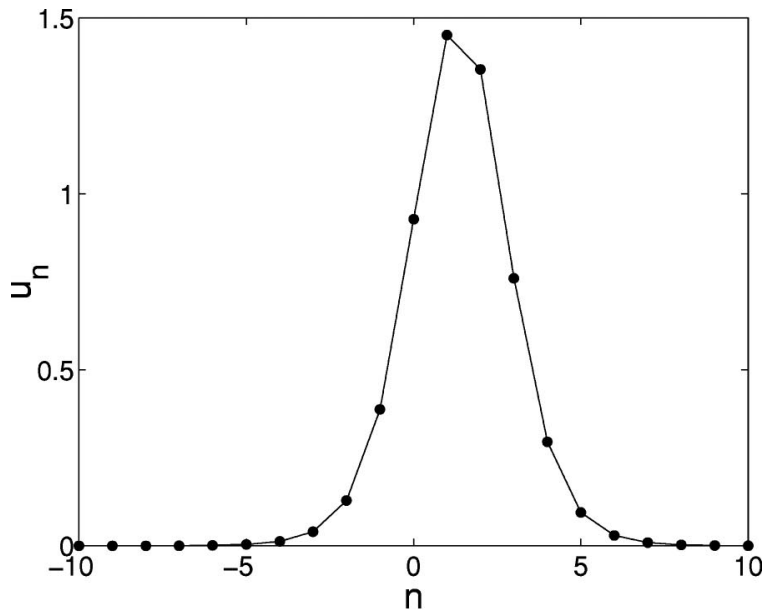

(a)

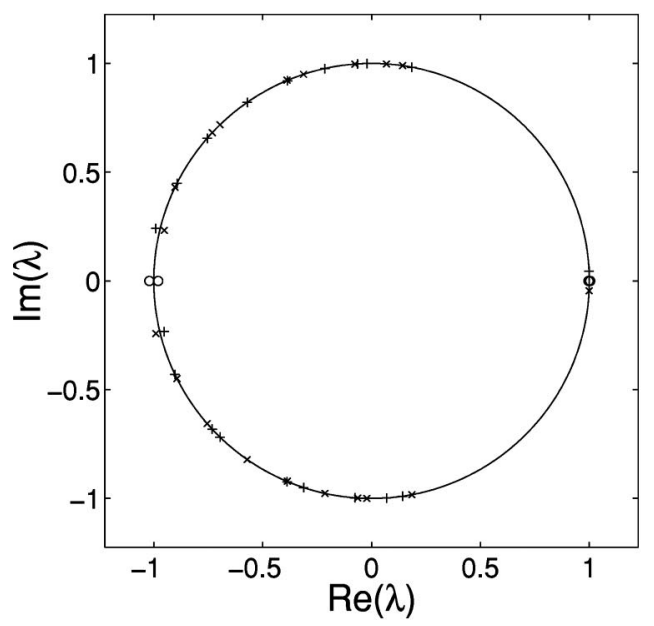

(b)

FIG. 6. (a) Spatial profiles of the breathers centered at $n=1$ for $\alpha=-0.003$ and the same characteristics to those of Figure 4. (a) shows the spatial profile of unstable solution; (b) represents the Floquet multipliers corresponding to this state. The instabilities that can be appreciated near $\theta=\pi$ are due to the collision of extended eigenmodes and disappear in the case of an infinite lattice.

$$
\alpha=\frac{\omega_{\mathrm{imp}}^{2}-\omega_{o}^{2}-2 C \pm \sqrt{\left(\omega_{\mathrm{imp}}^{2}-\omega_{o}^{2}\right)\left(\omega_{\mathrm{imp}}^{2}-\omega_{o}^{2}-4 C\right)}}{4 C},
$$

where the plus sign corresponds to $\omega_{\text {imp }}^{2}>\omega_{o}^{2}+4 C$ and the minus sign to $\omega_{\text {imp }}<\omega_{o}$.

Figure 2 shows the frequencies of the linear modes as a function of $\alpha=\gamma / C$ and the profile of the impurity modes. It can be observed that, for $\gamma>0$, the impurity mode is above the phonon band and, in consequence, has a zigzag vibrational pattern (i.e., a staggered mode). If $\gamma<0$, the mode is below the band and its sites oscillate in phase [20]. It is worth remarking that the impurity modes are antisymmetric modes whose central lattice site is at rest.

\section{STATIC RESULTS: STATIONARY BREATHERS AND THEIR BIFURCATIONS}

Discrete breathers can be calculated using well-known techniques based on the concept of continuation from the anticontinuous limit [23]. Furthermore, since the bending acts as an inhomogeneity, the system has lost its integer shift invariance and the properties of the breathers critically depend on the site where their center is located (at $\gamma=0$ ). This fact leads to the existence of saddle-node bifurcations through which some of the solutions can disappear. Similar bifurcations have been previously observed in bent chains of oscillators $[13,14]$. This has also been the main focus, for DNLS-type settings, of Ref. [8].

These bifurcations need a relatively high value of the coupling (sufficient cross talk between the neighboring sites) to be observed. Otherwise, breather solutions will exist for very large intervals of $\gamma$ in every site of the lattice. Hence we restrict ourselves to $C$ 's of the order of (typically) 0.1 to render these bifurcations tractable.

We now proceed to examine the bifurcation diagrams (as a function of $\alpha$ ) for both soft and hard on-site potentials and a harmonic intersite potential.

\section{A. Morse potential}

Figure 3 shows the bifurcation diagrams for the case of a Morse on-site potential and couplings $C=0.13$ and $C=0.26$ and frequency $\omega_{\mathrm{b}}=0.8$, and Fig. 4 shows the spatial profile of the solutions corresponding to a number of branches for $\alpha$ $=0.0005$.

For $C=0.13$, the branch corresponding to the breathers centered at $n=0$ merges with the breathers originally centered at $n=0.5$ at $\alpha \approx 0.00115$. The branches centered at $n$ $=1.5$ and $n=2$ disappear through a saddle-node bifurcation at $\alpha \approx 0.00057$. This phenomenon is also observed for the branches centered at $n=2.5$ and $n=3$ at $\alpha \approx 0.00674$. For $\alpha<0$ the annihilations are observed between different branches of solutions. In particular, the branches centered at $n=0.5$ and $n=1$ annihilate at $\alpha \approx-0.00049$ and the ones centered at $n=2$ and $n=2.5$ cease to exist through the saddlenode bifurcation occurring at $\alpha \approx-0.00120$. It should, however, be noted that some branches (see, e.g., the branch centered at $n=1$ for $\alpha>0$ or the one centered $n=-1.5$ for $\alpha<0$ ) never annihilate. It is also interesting to note that all of these branches of solutions are unstable, as both site-centered and bond-centered breathers are unstable in the straight chain (for this value of the NN coupling). However, the branch resulting when the centered at $n=0$ and $n=0.5$ merge is stable.

The branch centered at $n=1.5$ becomes stable for $\alpha$ $\leqq 0.002$. This change of stability has its origin in a pair of Floquet multipliers that collide in the unit circle at $\lambda=1$. Notice that this behavior, unlike the typical branches discussed above, does not involve a saddle-node bifurcation (or for that matter an exchange of stability with another static breather branch).

For $C=0.26$ the saddle-node bifurcations occur between different solutions from the case considered above. For $\alpha>0$, the branches centered at $n=0.5$ and $n=1$ collide and disappear at $\alpha \approx 0.00072$; the branches centered at $n=2$ and $n=2.5$ cease to exist at $\alpha \approx 0.00106$ while the ones centered at $n=3$ and $n=3.5$ terminate at $\alpha \approx 0.00342$. The branch centered at $n=0$ merges with the branch centered at $n=0.5$ for $\alpha<0$ and in particular for $\alpha \approx-0.00187$. 


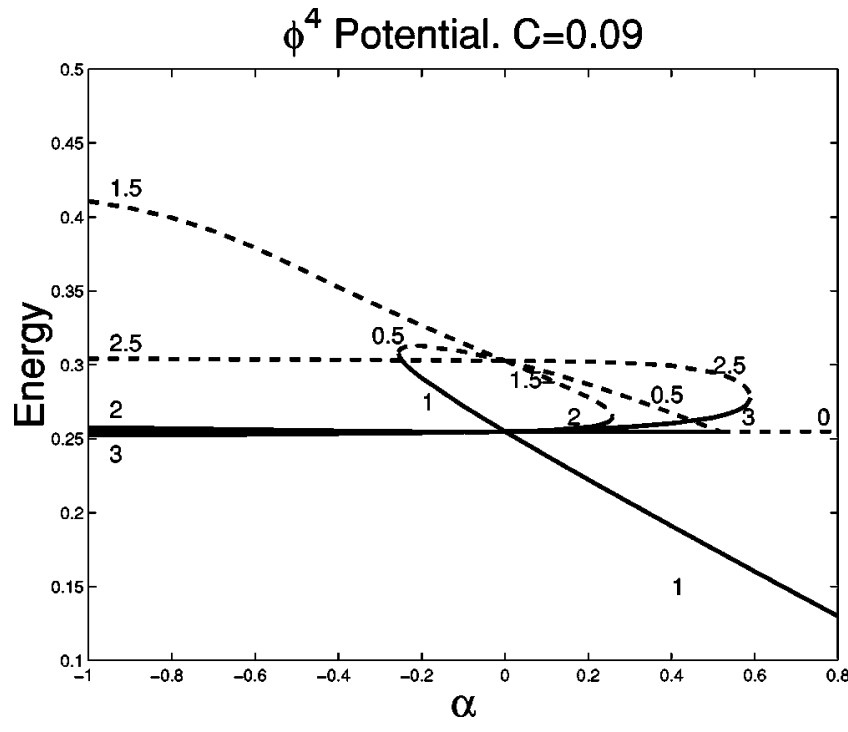

FIG. 7. Bifurcation diagram for the solutions with a $\phi^{4}$ on-site potential. The energy of the breathers is plotted as a function of the bending parameter $\alpha=\gamma / C$. The numbers indicate the site where the solutions are centered; the half-integer numbers actually correspond to two-site breathers, but the notation has been kept in consonance with Fig. 3. Stable solutions are represented by full lines whereas unstable solutions are represented by dashed lines.

In addition, the branches centered at $n=1.5$ and $n=2$ annihilate at $\alpha \approx-0.00204$ and the ones centered at $n=2.5$ and $n=3$ collide at $\alpha \approx-0.00157$. It is interesting to observe that similarly to what was found in Ref. [8], in a different setting, there is an asymmetric mode that persists for $\alpha<0$, whose energy is lower than the symmetric bend mode. Hence, for $\alpha<0$ in this case, we observe a symmetry breaking effect that leads to an asymmetric ground state of the system. Notice that similar asymmetric modes can be found (and identified to be potentially stable) in continuum models with localized impurities; see, e.g., Ref. [24].

It should be noted here that for this larger value of the coupling, the branches corresponding to site-centered solutions are stable for an infinite lattice, whereas the bondcentered solutions are unstable for the rectilinear chain. This stability is inherited by the branches of the bent chain. Motivated by the change of stability at the "unbent" limit $(\gamma$ $=0$ ), occurring as a function of $C$ (e.g., notice above the different stability of this limit for $C=0.13$ and $C=0.26$ ), we briefly study the stability of the rectilinear chain limit, as a function of $C$. To illustrate the stability of the one-site and two-site modes in the rectilinear chain (discussed in part in Ref. [16]), we examine their Floquet multipliers, both by means of the argument angles as well as giving their absolute values in Fig. 5. Notice, in particular, for the one-site modes that there are two inversions of stability, namely one at $C$ $\approx 0.1297$ and another for $C \approx 0.2382$. For $C>0.2382$, the one-site modes are stable but with interspersed sizedependent windows of instability (see, e.g., Refs. [25-27]).

Finally, let us mention that the branch that originates by the merging of the $n=0$ and $n=0.5$ branches is unstable. Figure 6 shows an example of the spatial profile and the Floquet eigenvalues of an unstable state for $\alpha<0$, namely a breather (originally) centered around $n=1$. Notice however that the instability in this case is a result of the finite size of the lattice.

\section{B. $\phi^{4}$ potential}

The most significant difference of the $\phi^{4}$ case with respect to the Morse one is that in the former (i.e., for a hard potential), the breathers bifurcate from the top of the continuous spectrum and as a result the corresponding spatial profiles are staggered [1]. Hence, in this case the main solutions of interest are one-site or two-site breathers with the adjacent sites oscillating in antiphase.

The hard $\phi^{4}$ potential has been considered with a coupling constant $C=0.09$ and solutions with frequency $\omega_{\mathrm{b}}$ $=1.2$. For these parameter values (notice that $C$ is comparable to the values used in Fig. 3), the saddle-node bifurcations of interest occur at considerably larger values of $\alpha$ for the present model in comparison to the Morse case; see, e.g., Fig. 7. In particular, the branch of the on-site breathers centered at $n=0$ merges with the intersite branch centered at $n$ $=0.5$ at $\alpha \approx 0.516$. Similarly, the on-site solutions centered at $n=2$ and $n=3$ collide (and disappear) with the corresponding intersite ones centered at $n=1.5$ and $n=2.5$ at $\alpha \approx 0.258$ and $\alpha \approx 0.590$, respectively. However, in this case, the branch centered at $n=1$ becomes the asymmetric, lowest energy, stable ground state that persists for any value of $\alpha$. For $\alpha<0$, the on-site breathers centered at $n=1$ annihilate with the intersite ones centered at $n=0.5$ at $\alpha \approx-0.255$. Notice that here also on-site solutions are stable, while intersite ones are unstable for $\gamma=0$, but the branch emerging from the $n=0$ and $n=0.5$ is unstable.

Some of the breather solutions for $\alpha=0.1$ are shown in Fig. 8 and the profile of the ground state for $\alpha>0$ together with its Floquet eigenvalues in Fig. 9.

\section{Instabilities: Switching and breather mobility}

As explained above, the ground state is not always the breather centered at $n=0$. In particular, for a Morse potential, $C=0.26, \omega_{\mathrm{b}}=0.8$ and $|\alpha|$ large enough, the ground state for $\alpha<0$ is the breather centered at $n=1$ (at least in the infinite domain limit, since in the finite domain case, it may be unstable as shown in Fig. 6). On the other hand, for $\alpha>0$ the ground state is still the breather at $n=0$. Thus, if an unstable solution (say, the $n=0$ centered for $\alpha<0$ or the $n=1.5$ for $\alpha>0$ ) is perturbed, it is likely to switch to the ground state, in a similar fashion to the phenomenon observed in Ref. [13]. However, as Fig. 10 shows, this may not always be the case. In particular, the breather oscillates between the sites $n=0$ and $n=3.5$ (i.e., in the vicinity of the stable ground state) for $\alpha<0$, while for $\alpha>0$, it is set in motion. A possible explanation relies on the fact that the eigenmode leading to the instability is actually a pinning mode, which is responsible for breather mobility (see also Sec. V). Hence a perturbation with the appropriate sign (i.e., causing the breather to move away from the ground state of $n=0$ rather than toward it) along this eigendirection can lead to breather motion. 


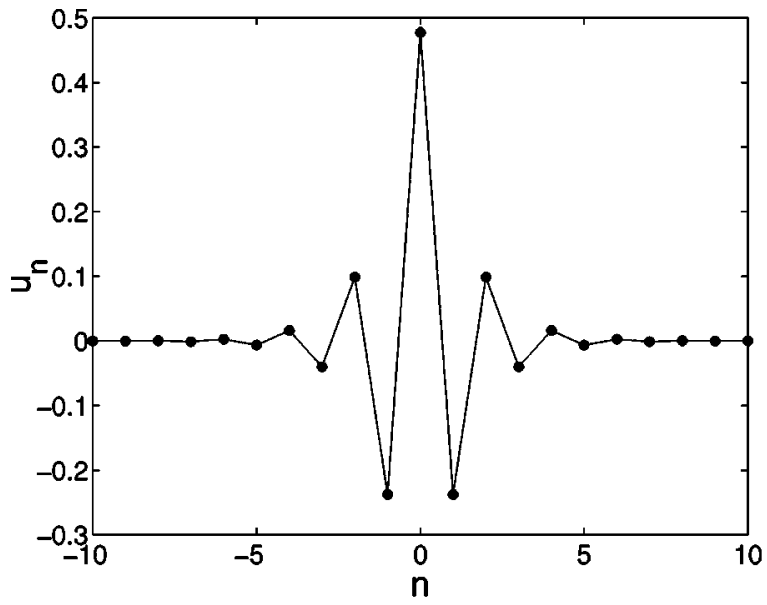

(a)

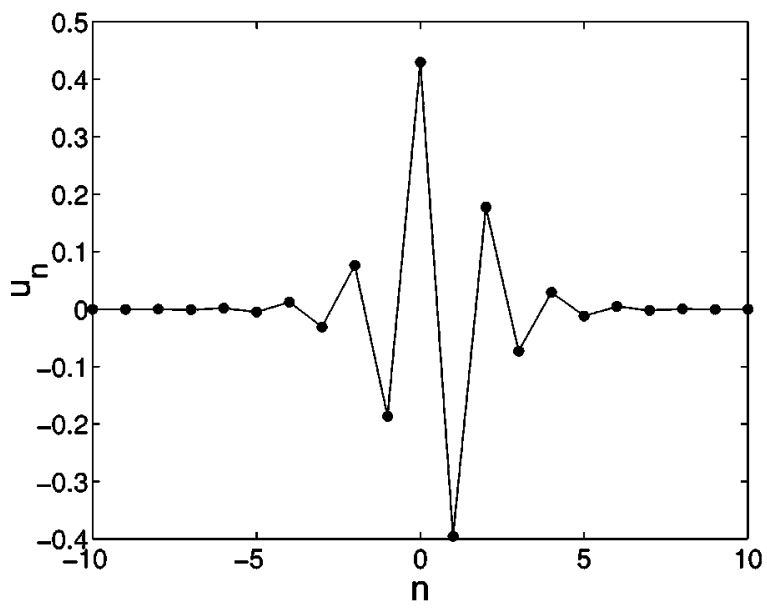

(b)

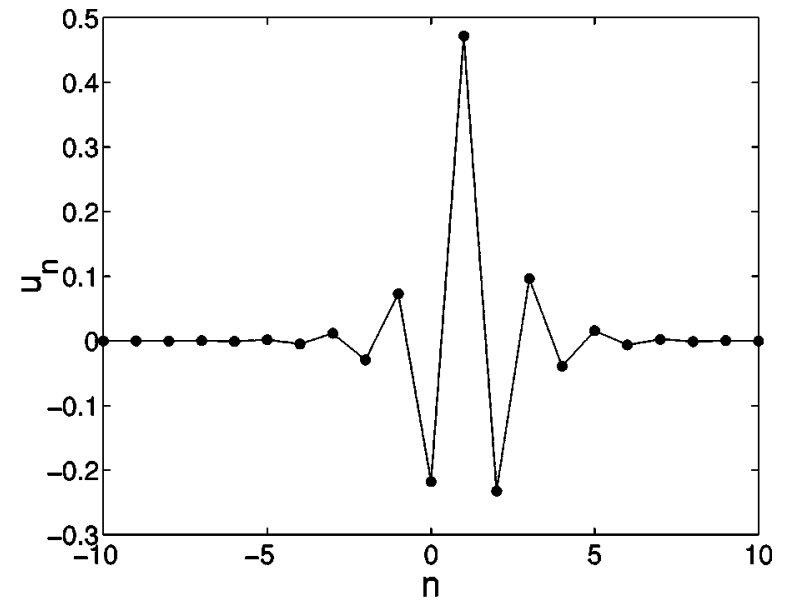

(c)

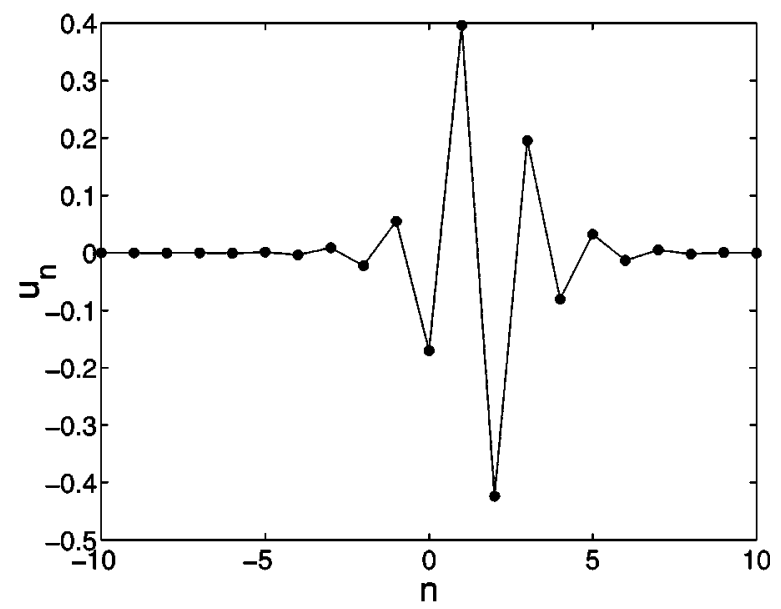

(d)

FIG. 8. Spatial profiles of the breathers centered at $n=0$ (a), $n=0.5$ (b), $n=1$ (c) and $n=1.5$ (d) for $\alpha=0.1$. The results correspond to a chain of oscillators with an on-site $\phi^{4}$ potential with breather frequency $\omega_{\mathrm{b}}=1.2$ and coupling constant $C=0.09$.

For a $\phi^{4}$ potential, $C=0.09, \omega_{\mathrm{b}}=1.2$, and $|\alpha|$ large enough, the ground state for $\alpha>0$ is the breather centered at $n=1$, while for $\alpha<0$, it is not well defined.

Figure 11 shows the evolution of a two-site breather centered at $n=2$ with $\alpha=0.5$. It is observed that the breather can switch to the one-site breather centered at $n=1$, i.e., the ground state. In this case, no moving localized excitations arise, but rather the previously suggested switch occurs. This may be attributed to the fact that for the hard $\phi^{4}$ potential, there do not exist pinning modes that could potentially lead to breather mobility (see also Ref. [28]).

\section{DYNAMIC RESULTS: MOVING BREATHERS AND BREATHER-BEND INTERACTIONS}

In this section we examine how the local geometry of the bend can influence the motion of a breather in its vicinity. In particular, the moving breathers are launched towards the bend vertex following the marginal mode method [28]. The latter consists of adding to the velocities of the static breather a perturbation of magnitude $\lambda$, collinear to the direction of a specific linear localized mode, namely the eigenmode corre- sponding to translation. Then, the kinetic (translational) energy of the breather can be defined as $K=\lambda^{2} / 2$. As Ref. [15] shows, the moving breather can be described as a quasiparticle of mass $m^{*}$ defined through the relation $K=\lambda^{2} / 2$ $=m^{*} v^{2} / 2$, with $v$ being the translational velocity of the breather. The mass is a measure of the inertia of the breather to external forces.

Another useful concept for our study is the breather energy center, which is a measure of the position of the localized structure. It is defined through the relation $X$ $=\left(\Sigma_{n} n e_{n}\right) / E$, i.e., as the center of mass with respect to $e_{n}$, the energy density at the $n$th site. $E=\Sigma_{n} e_{n}$ is the total energy of the breather [16].

The local "coupling inhomogeneity" induced by the bend should result in features similar to those observed in Ref. [20]. In particular, for a soft potential, if $\alpha>0$, the bending should act, in principle (see also below), as a potential barrier, i.e., the breather can cross the vertex as long as its translational energy is above a threshold value. If this condition is satisfied, the breather decelerates when crossing the bend, but eventually recovers its initial velocity. If, on the other hand, $\alpha<0$, then the corresponding behavior is more complex: for small values of $|\alpha|$, the bend acts as a potential 


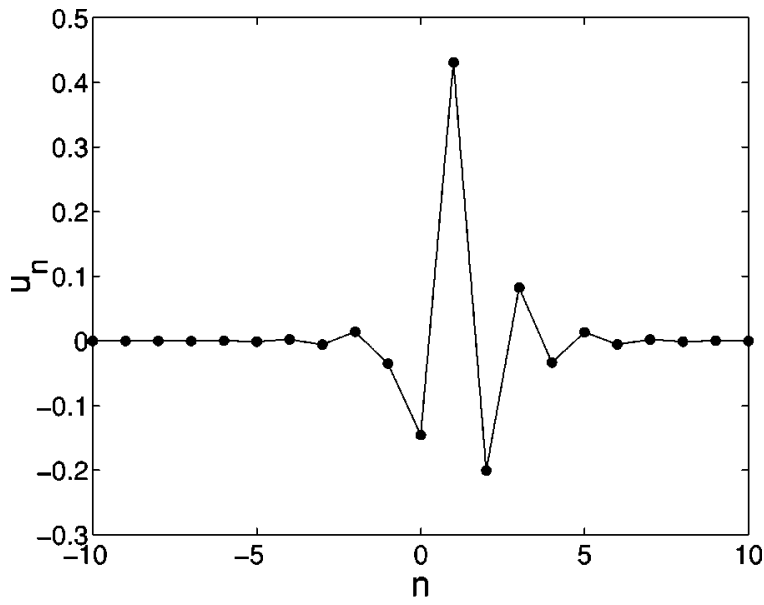

(a)

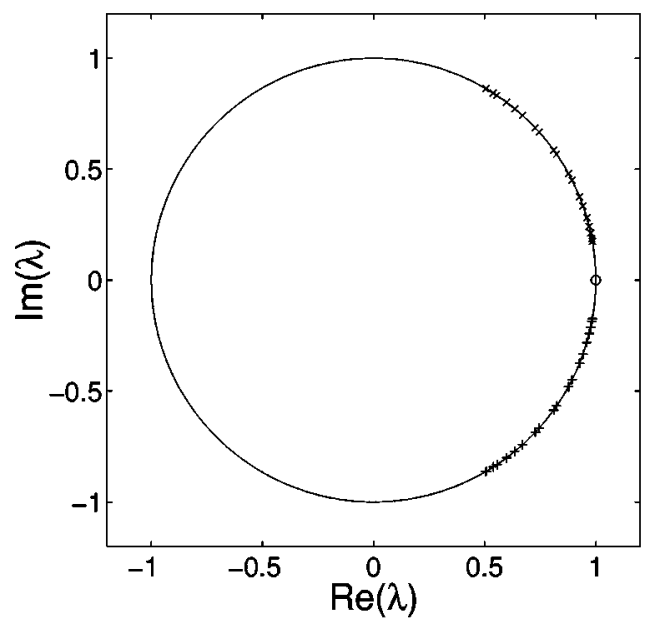

(b)

FIG. 9. (a) Spatial profiles of the breathers centered at $n=1$ for $\alpha=0.5$ and the same characteristics to those of Fig. 8. This solution corresponds to the stable ground state. (b) represents the Floquet eigenvalues of this solution.

well, accelerating a moving breather approaching it. However, for larger values of the parameter, trapping and even reflection become possible. For a hard potential, such as $\phi^{4}$, the behaviors for $\alpha>0$ and $\alpha<0$ are reversed [29].

We will focus on the $\alpha>0$ case and consider, as before, a chain of oscillators with (1) the Morse on-site potential, as well as one with (2) the $\phi^{4}$ on-site and intersite potential. Thus, for the case of the Morse (soft) potential, the bending will act as a potential barrier, while for the $\phi^{4}$ (hard) potential, the well/trapping/reflection regimes will be examined.

\section{A. Morse chain: Bend-induced potential barrier}

As mentioned earlier, for a soft on-site potential and $\alpha>0$, the bending acts as a potential barrier, i.e., if the translational energy of the breather $K$ is above a threshold value $U_{c}$, the breather crosses the bending. Otherwise, the breather is reflected. Figure 12 shows the time evolution of a reflected (top panel), a refracted (middle panel), and that of a trapped
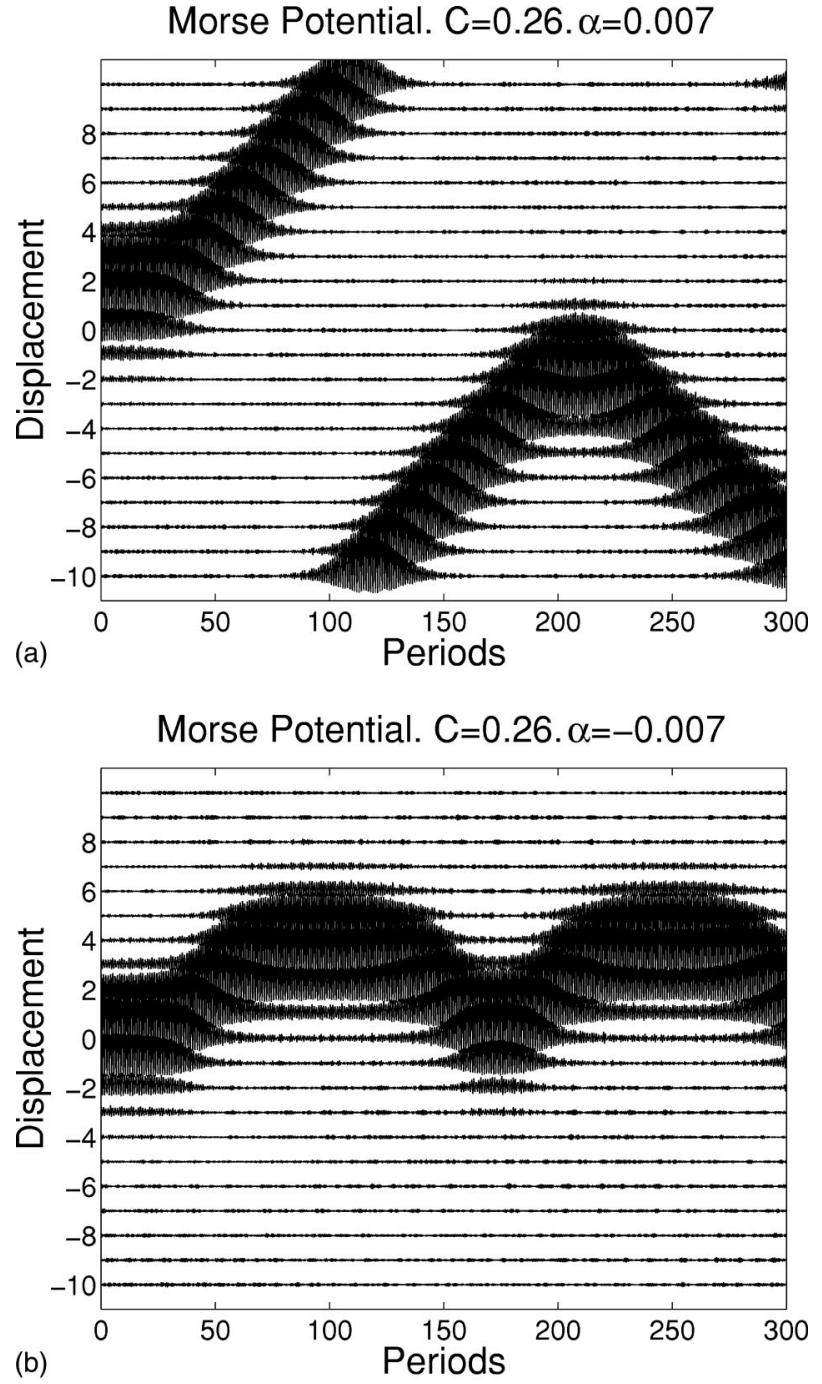

FIG. 10. Time evolution of an unstable breather slightly perturbed for $\alpha>0$ (a) and $\alpha<0$ (b).

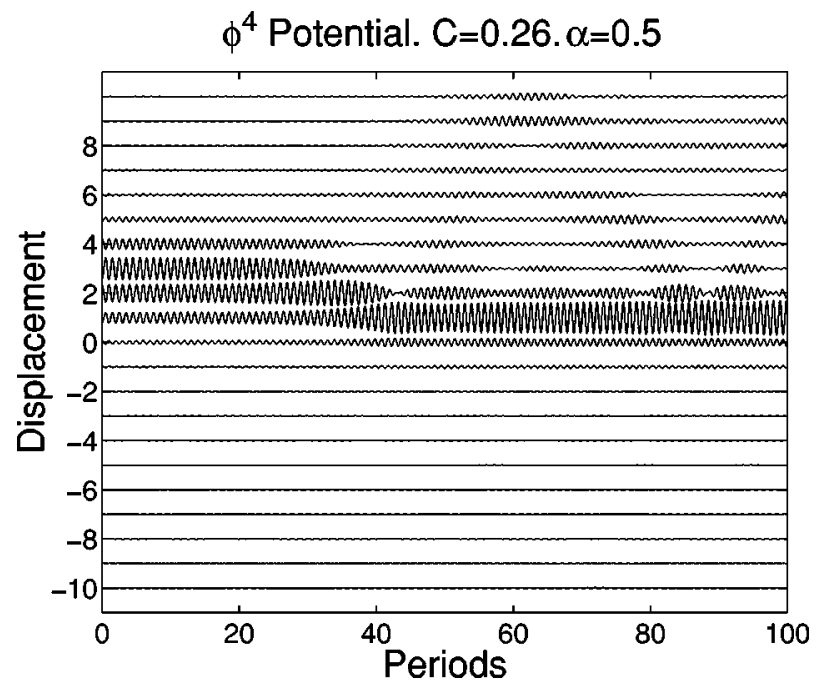

FIG. 11. Time evolution of an unstable breather slightly perturbed for $\alpha>0$. 

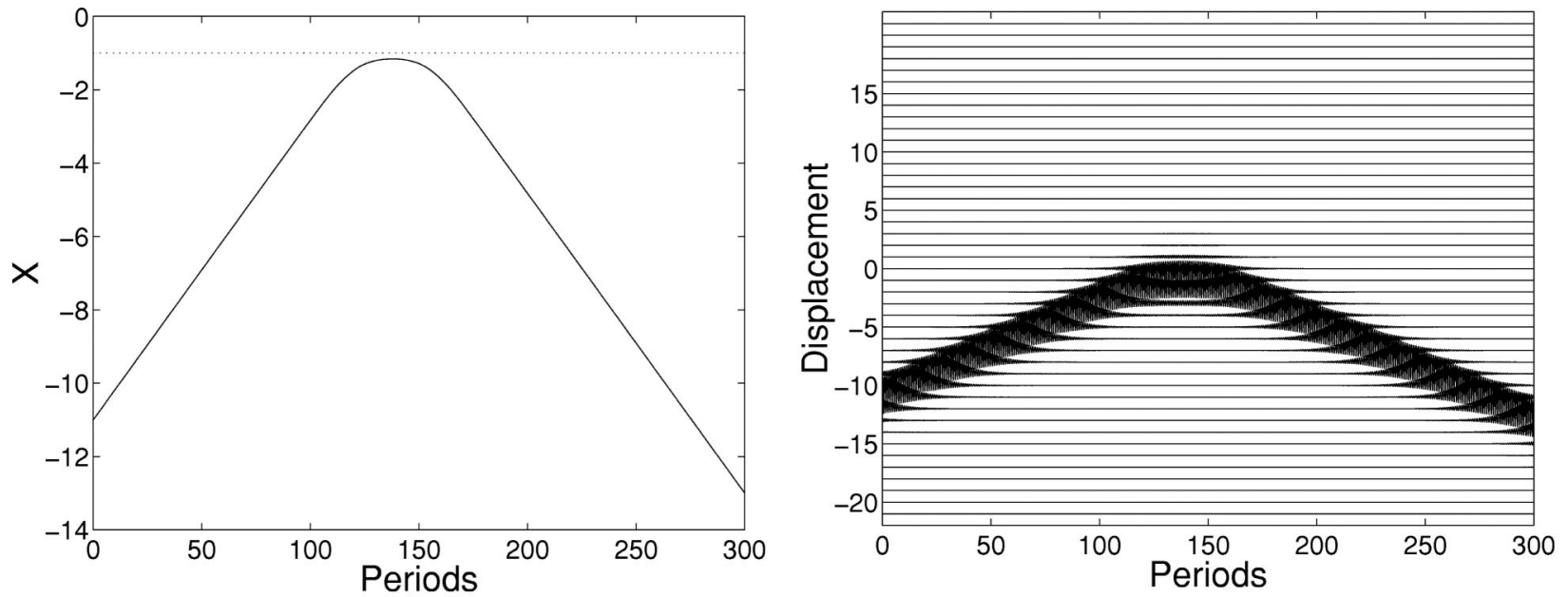

(a)

(b)
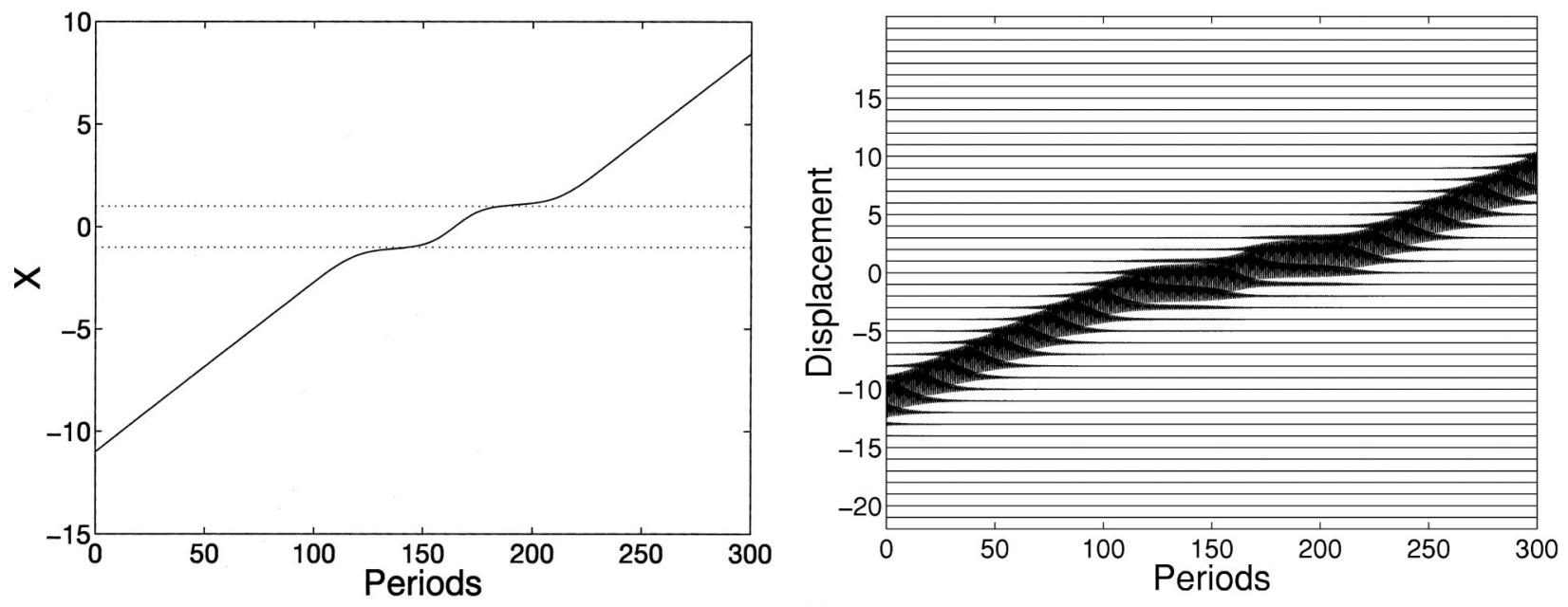

(c)

(d)
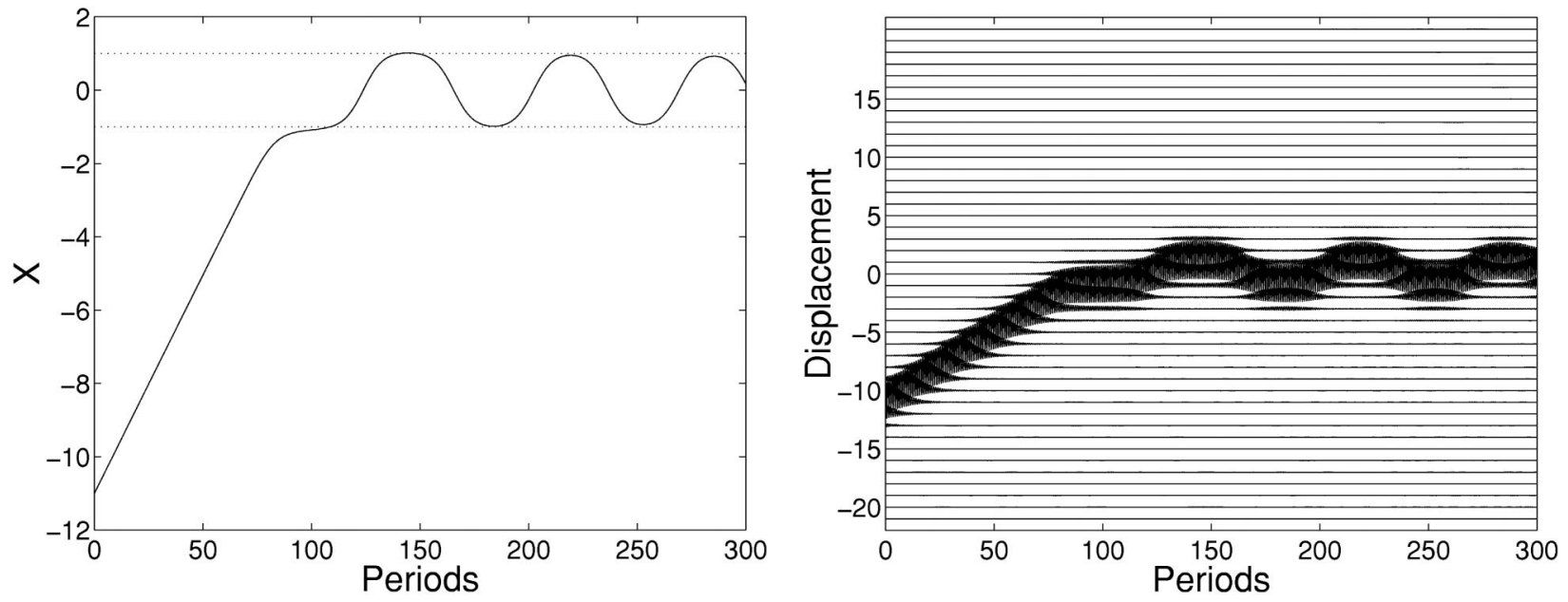

(f)

FIG. 12. Evolution of the energy center (left) and the moving breather (right) for a Morse on-site potential with parameters $\omega_{\mathrm{b}}=0.8$ and $C=0.13$. Figures (a) and (b) correspond to a reflection ( $\alpha=0.008$ and $K=0.00174)$, (c) and (d) to a refraction $(\alpha=0.008$ and $K=0.00180)$ and (e) and (f) to a refraction through the site $n=-1$ and a reflection at $n=1$ leading to a trapping $(\alpha=0.008$ and $K=0.00392)$. 


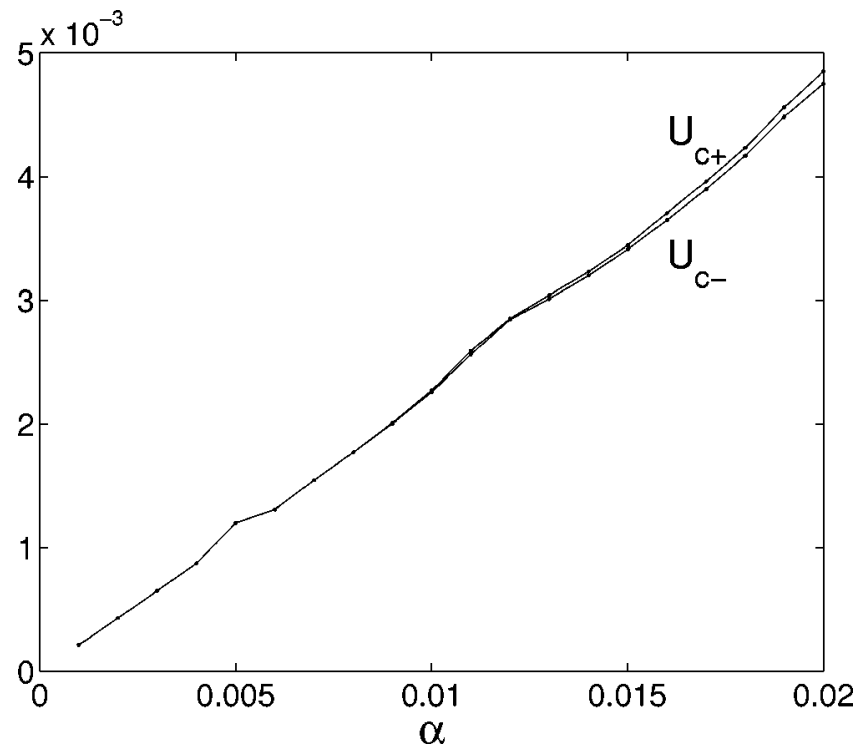

FIG. 13. Dependence of the critical energy $U_{c}$ on $\alpha$ for a Morse on-site potential with parameters $\omega_{\mathrm{b}}=0.8$ and $C=0.13$. Note that for sufficiently large $\alpha$, the curve separates in two parts corresponding to $U_{c+}$ and $U_{c-}$ (see also the relevant discussion in the text).

breather (bottom panel). In the top panel (for $K<U_{c}$ ), the breather is immediately reflected from the potential barrier, emerging with (approximately) the same speed, but propagating in the opposite direction. In the middle panel of the figure, it can be observed that for $K>U_{c}$ the breather decelerates when it approaches the $n=-1$ site, and accelerates when it crosses this point until it reaches the bend vertex ( $n=0$ site); it decelerates again when approaching the $n=1$ site, accelerating when it crosses this point.

Figure 13 shows the critical energy $U_{c}$ necessary to cross the bend. An important point to indicate is the existence of two distinct values of the critical energy $U_{c \pm}$, which correspond, respectively, to the kinetic energy threshold for crossing the site $n= \pm 1$. When $U_{c-}<U_{c+}$ holds, for intermediate values of $U_{-} \leqslant K<U_{+}$, the loss of energy due to phonon radiation in the breather-bend interaction disallows the moving breather from crossing the bend. Hence, if the two thresholds are different, the breather can be trapped oscillating between the $n=-1$ and $n=1$ sites; an example is shown in the bottom panel of Fig. 12. As is naturally expected, the energy loss decreases when $\alpha$ is small (i.e., as we approach the rectilinear, integer-shift invariant chain), and $U_{c-}=U_{c+}$ is practically fulfilled in this parameter range, e.g., for $\alpha<0.01$ in Fig. 13.

The potential barrier $U=U(X)$ can be calculated using the procedure described in Ref. [15]: if $K<U_{c}$, the value of $K$ is fixed and $X_{o}$ is the point where the breather is reflected (turning point), then $K=U\left(X_{o}\right)$.

If, on the other hand, $K>U_{c}$, the barrier can be calculated through the formula

$$
U(X)=K\left[1-\left(\frac{v(X)}{v_{o}}\right)^{2}\right],
$$

where $v_{o}$ is the initial translational velocity of the breather and $v(X)$ is its (numerically computed) velocity at the point

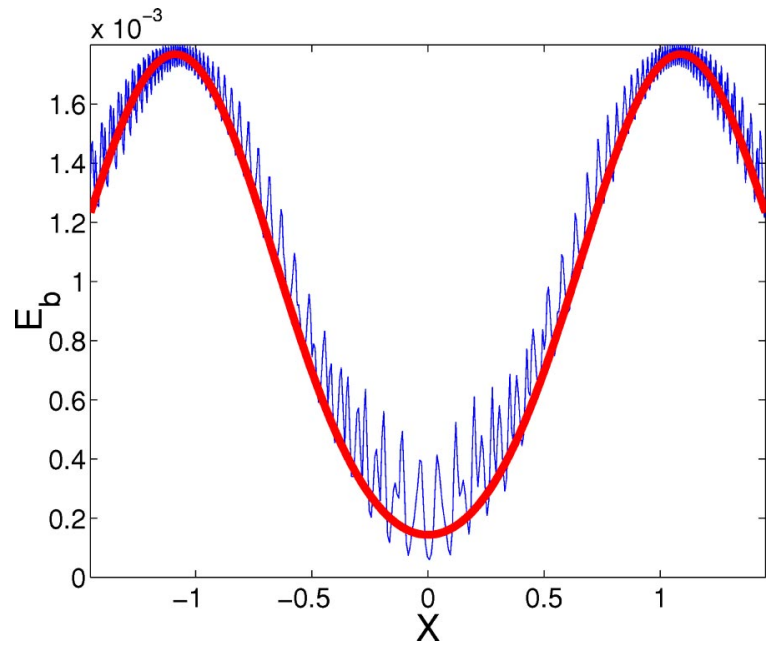

FIG. 14. Potential barrier experienced by the moving breather in the chain with a Morse on-site potential, when it reaches the bending point. Parameters of the breather are $\omega_{\mathrm{b}}=0.8, C=0.13$, and $\gamma$ $=0.08$. The thick line corresponds to the fit to Eq. (8) and the slim line to Eq. (6) for $K=0.0018$. The oscillations of the last curve are due to the nonuniformity of the translational velocity of the breather. Parameters of the fitting to Eq. (8) are $a=0.001768, b$ $=2.7068$, and $\delta=0.08754$.

$X$. If the mass of the breather is constant, the barrier calculated for $K<U_{c}$ and $K>U_{c}$ should coincide, except for the oscillations that appear in the second case with respect to the first one. The origin of these oscillations relies on the nonuniformity of the instantaneous translational velocity of the breather due to the discreteness of the system. Thus, to obtain the actual shape of the barrier, the turning point approach should be used.

However, this method can only be applied in our case for $X<-1$ (and hence by symmetry for $X>1$ ) as for $K<U_{c}$, the breather cannot cross over to the region $-1<X<1$. In order to overcome this drawback, we have approximated the potential barrier created by a single inhomogeneity with a Gaussian shape: $U(X)=a \exp \left[-b\left(X-X_{o}\right)^{2}\right]$, where $X_{o}$ is the point where the maximum of the barrier is located (which need not be the inhomogeneity point) $[8,26,30]$. In our bend setting, there are two points where the equation is inhomogeneous with respect to the rest of the chain. Hence, it is natural to approximate the actual barrier with the linear superposition of two Gaussian barriers, i.e.,

$$
\begin{gathered}
U(X)=a \exp \left[-b\left(X-X_{-1}\right)^{2}\right]+\exp \left[-b\left(X-X_{1}\right)^{2}\right], \\
x_{ \pm 1}= \pm(1+\delta),
\end{gathered}
$$

with $\delta$ being the difference between the turning point at $K$ $\approx U_{c}$ and the "impurity site"; e.g., for a breather launched from the left of the bending, $\delta=X\left(U_{c}\right)+1$. Thus, Eq. (7) becomes

$$
U(X)=2 a \exp \left(-b X^{2}\right) \exp \left[-b(1+\delta)^{2}\right] \cosh [2 b X(1+\delta)] .
$$




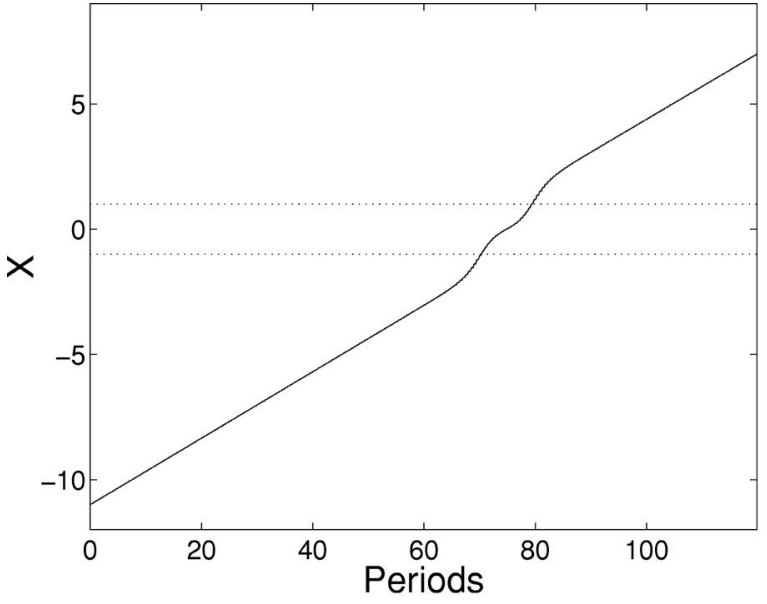

(a)

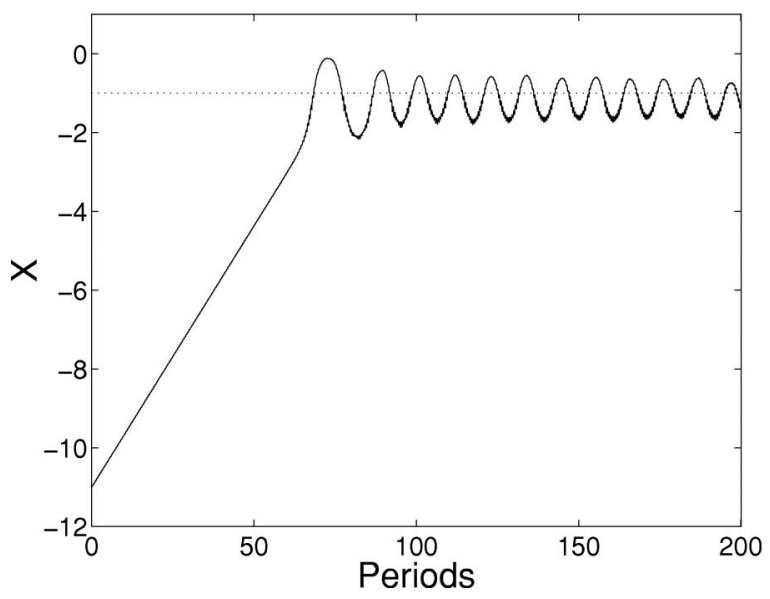

(c)

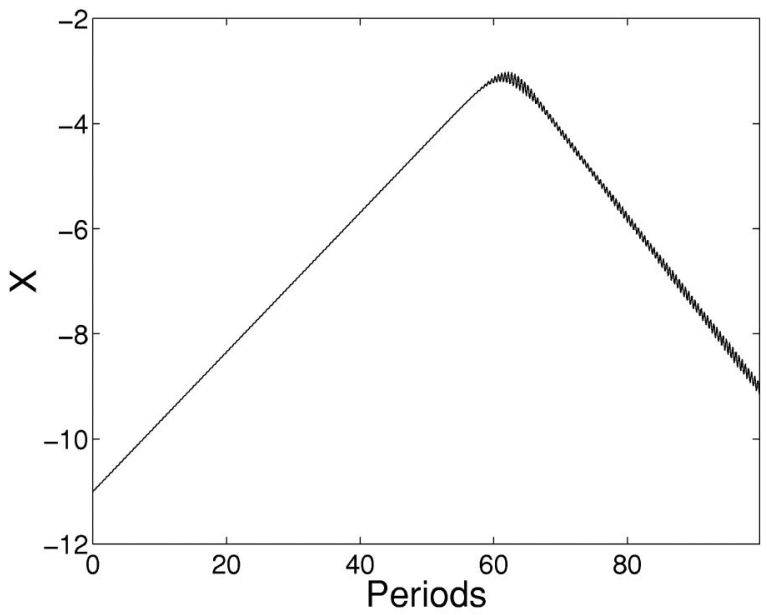

(e)

FIG. 15. Evolution of the energy center (left) and the moving breather (right) for $\phi^{4}$ on-site and interaction potentials with parameters $\omega_{\mathrm{b}}=3$ and $C=0.5352$. (a) and (b) correspond to a refraction $(\alpha=0.2)$, (c) and (d) to a trapping $(\alpha=0.5)$, and (e) and (f) to a reflection ( $\alpha$ $=4)$.

Figure 14 shows the barrier calculated using Eqs. (6) and (8). Parameters $a, b$, and $\delta$ are chosen through the Gaussian fit of the barrier points for $|X|>1$. Comparing the fitted profile of Eq. (8) with the one obtained for a supercritical case of $K>U_{c}$ indicates very good agreement, which in turn supports the assumption of Eq. (7).

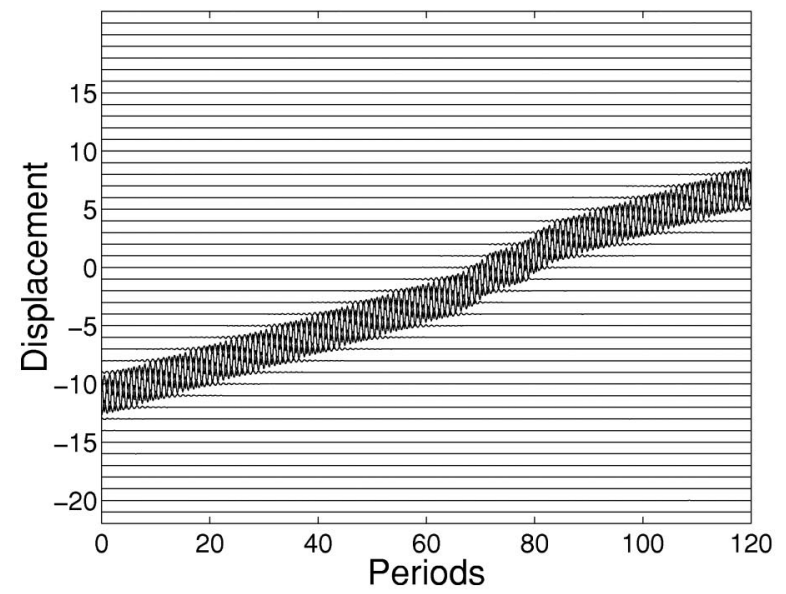

(b)

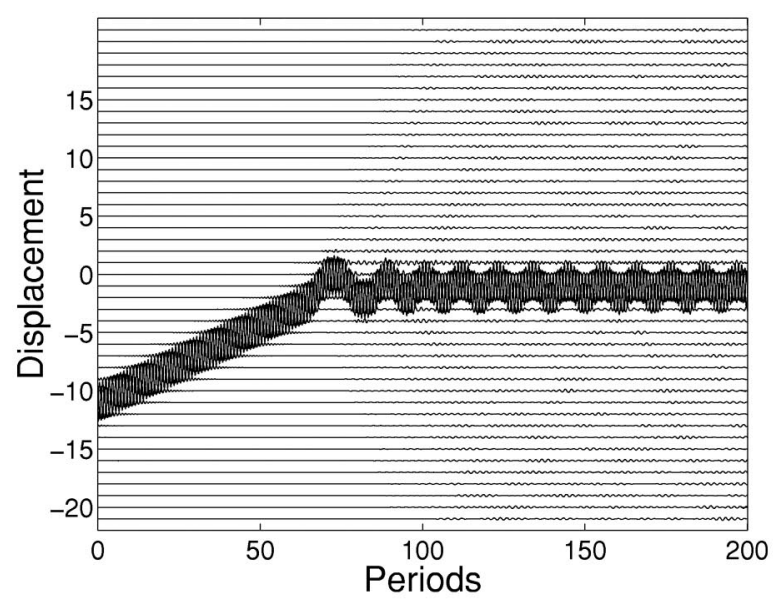

(d)

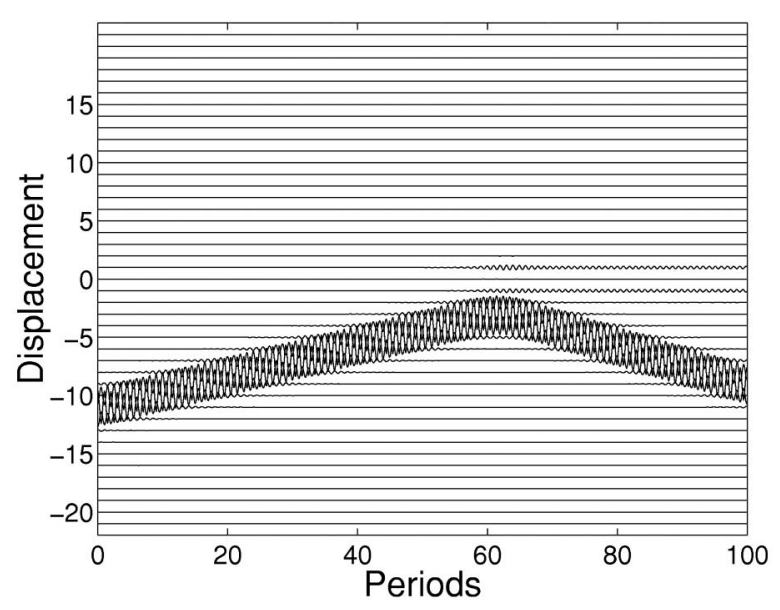

Notice, however, that the such a restricted one-degree-offreedom picture cannot capture phenomena such as emission of radiative small amplitude waves during the crossing of the bend, and consequently cannot display a phenomenology as rich as the original problem (e.g., it cannot exhibit the trapping-type phenomena discussed above). 


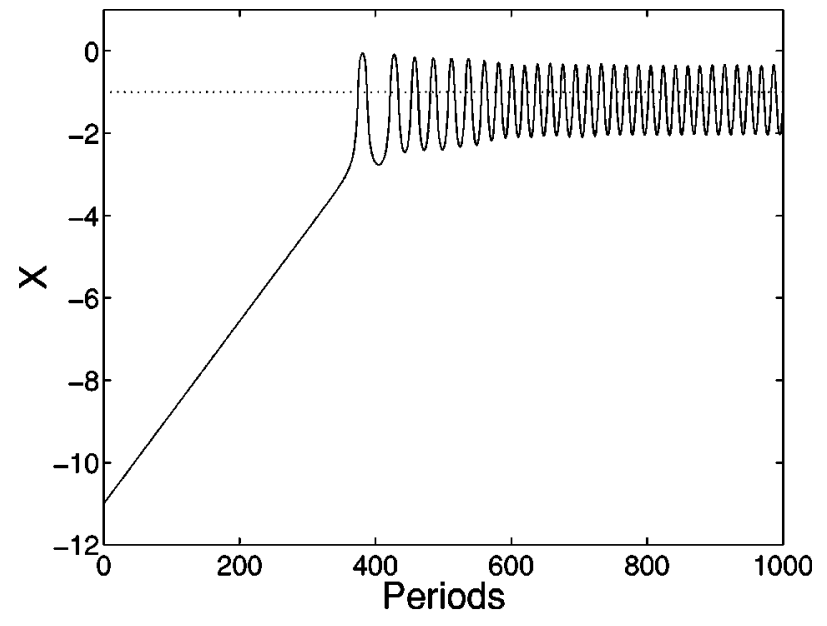

(a)

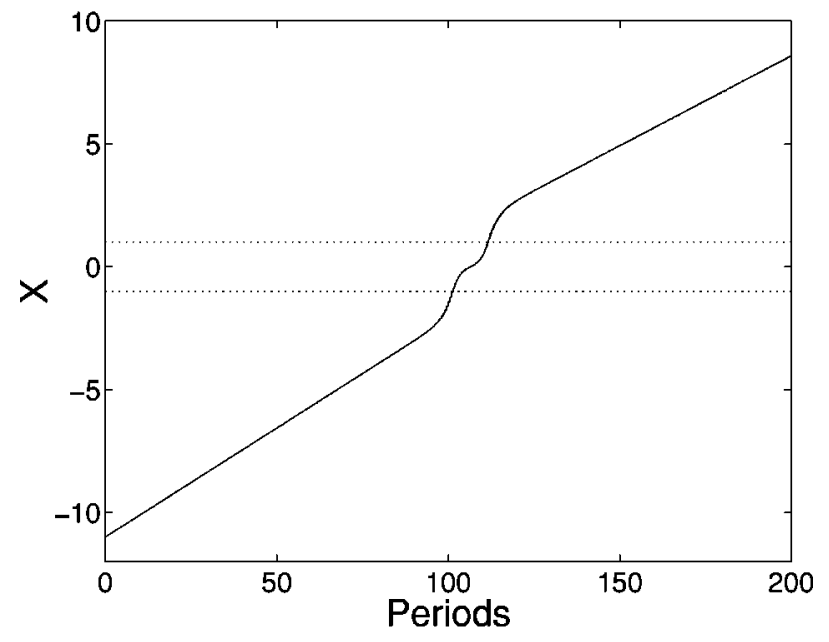

(b)

FIG. 16. Evolution of the energy center for a $\phi^{4}$ on-site and interaction potentials with parameters $\omega_{\mathrm{b}}=3, C=0.5352$ and a fixed value of $\alpha=0.3$. (a) corresponds to a trapping case $(K=0.00125)$ and (b) to a well regime $(K=0.02)$.

\section{B. $\phi^{4}$ chain: Well/trapping/reflection regimes}

For the case of a hard potential with $\alpha>0$ three different behaviors can be observed when $\alpha$ increases (see Fig. 15).

(1) For small values of $\alpha$, the breather accelerates when reaching the bend points, and eventually emerges from the bend recovering a constant velocity (but not the same as the initial one due to radiative emissions). That is, the bending acts as a potential well.

(2) For larger values of $\alpha$, the breather is trapped at the $n=-1$ site.

(3) For even larger values of $\alpha$, the breather is reflected and the particle at the vertex remains excited. This phenomenon can be also called partial trapping, since a fraction of the original breather energy remains localized at the bend.

(4) Finally, beyond a third critical point for $\alpha$, the breather is reflected from the bend, and, in a different fashion to the barrier case, the breather is not refracted for any velocity.

It should be noted that the boundaries between partial trapping to reflection and well to trapping regimes are not well defined. In the first case, the amplitude of the vertex excitation decreases when $|\alpha|$ increases. Due to this fact, it is difficult to establish the borderline between those regimes. In the second case, the breather is trapped if its translational energy is below a threshold value. However, for energies above this value, the bend acts as a potential well. Thus, the trapping takes place without need of energy loss when the breather crosses the $n=-1$ site, that is, it occurs as long as the translational energy is below the threshold commented above. These facts are illustrated in Fig. 16.

\section{CONCLUSIONS}

In this paper, we have introduced a discrete Klein-Gordon chain model variant, emulating the existence of a geometric bend at a lattice site.

We have examined the static properties of the localized in space, time periodic solutions that exist in this setting, starting from the limit where the chain is rectilinear. In the latter case, from the integer shift invariance and numerous earlier studies [1], we know that site-centered and bond-centered solutions will exist and their stability can be established. Hence, using continuation from the "straight" limit into the "bent" regime, we are able to trace the branches of corresponding solutions and identify their saddle-node bifurcations leading to the termination of the corresponding branches. However, we have also identified symmetry breaking effects in which one of the asymmetric branches does not collide with one of its neighboring counterparts to terminate its existence, but rather survives throughout the bendparameter strength continuation, and becomes the lowest energy state of the lattice. Excitation of unstable bend modes in such cases will lead to trapping around (i.e., switching to) such an asymmetric state, but in the presence of pinning modes, moving breathers may result. Hence, we conclude that sufficiently strong bends may favor an asymmetric localization of energy, with respect to the bend center.

We have also studied the dynamic properties of the interaction of such bends with moving localized modes, when the latter are scattered off of a bend. We have found that for soft potentials, the bend operates as a potential barrier allowing transmission for supercritical and reflection for subcritical values of the localized excitation's initial speed. However, there can also be narrow intervals of trapping (not captured by the potential barrier picture). On the other hand, for hard potentials, a more complex and partly initial condition (i.e., kinetic energy) dependent picture emerges. For small bend strengths, the inhomogeneity acts as a potential well, but for higher ones it can lead to trapping and even to reflection.

In this numerical study, we have mainly aimed at presenting the relevant phenomenology, classifying it for KleinGordon chains with different potentials, and providing a qualitative explanation of the relevant findings. It would naturally be of interest to attempt to obtain more quantitative, theoretically predicted, estimates for the transitions observed herein. A variational viewpoint may prove to be useful in this context. 


\section{ACKNOWLEDGMENTS}

The support of Grant No. NSF-DMS-0204585, a University of Massachusetts Faculty Research Grant, and of the Eppley Foundation for Research is gratefully acknowledged
(P.G.K.). J.C. acknowledges an FPDI grant from 'La Junta de Andalucía' and partial support under the European Commission RTN project LOCNET, Grant No. HPRN-CT-199900163.
[1] S. Aubry, Physica D 103, 201 (1997); S. Flach and C. R. Willis, Phys. Rep. 295, 181 (1998); Physica D 119 (1999), special issue, edited by S. Flach, R. S. MacKay; P. G. Kevrekidis, K. Ø. Rasmussen, and A. R. Bishop, Int. J. Mod. Phys. B 15, 2833 (2001); Chaos13, 586 (2003), special issue, edited by Yu. S. Kivshar and S. Flach.

[2] See, e.g., A. A. Sukhorukov et al., IEEE J. Quantum Electron. 39, 31 (2003); H. S. Eisenberg et al., J. Opt. Soc. Am. B 19, 2938 (2002); U. Peschel et al., ibid. 19, 2637 (2002).

[3] A. Trombettoni and A. Smerzi, Phys. Rev. Lett. 86, 2353 (2001); F. Kh. Abdullaev et al., Phys. Rev. A 64, 043606 (2001); F. S. Cataliotti et al., Science 293, 843 (2001); A. Smerzi et al., Phys. Rev. Lett. 89, 170402 (2002).

[4] E. N. Pelinovsky and S. K. Shavratsky, Physica D 3, 410 (1981).

[5] P. Binder et al., Phys. Rev. Lett. 84, 745 (2000); E. Trías, J. J. Mazo, and T. P. Orlando, ibid. 84, 741 (2000).

[6] M. Peyrard, and A. R. Bishop, Phys. Rev. Lett. 62, 2755 (1989); T. Dauxois, M. Peyrard, and A. R. Bishop, Phys. Rev. E 47, R44 (1993); 47, 684 (1993); M. Peyrard et al., Physica D 68, 104 (1993); A. Campa and A. Giansanti, Phys. Rev. E 58, 3585 (1998).

[7] S. F. Mingaleev, Yu. S. Kivshar, and R. A. Sammut, Phys. Rev. E 62, 5777 (2000); S. F. Mingaleev and Yu. S. Kivshar, Phys. Rev. Lett. 86, 5474 (2001); D. N. Christodoulides and N. K. Efremidis, Opt. Lett. 27, 568 (2002); S. F. Mingaleev and Yu. S. Kivshar, Opt. Photonics News 13, 48 (2002).

[8] Yu. S. Kivshar, P. G. Kevrekidis, and S. Takeno, Phys. Lett. A 307, 287 (2003).

[9] Y. B. Gaididei, S. F. Mingaleev, and P. L. Christiansen, Phys. Rev. E 62, R53 (2000).

[10] P. G. Kevrekidis, B. A. Malomed, and A. R. Bishop, Phys. Rev. E 66, 046621 (2002).

[11] I. Bena, A. Saxena, and J. M. Sancho, Phys. Rev. E 66, 045603 (2003); I. Bena et al., ibid. 67, 037601 (2003).

[12] M. Ibanes, J. M. Sancho, and G. P. Tsironis, Europhys. Lett. 57, 697 (2002); Phys. Rev. E 65, 041902 (2002).
[13] J. F. R. Archilla et al., J. Phys. A 34, 6363 (2001).

[14] J. F.R. Archilla, P. L. Christiansen, and Yu. B. Gaididei, Phys. Rev. E 65, 016609 (2001).

[15] J. Cuevas et al., Phys. Lett. A 299, 221 (2002).

[16] J. Cuevas et al., Physica D 163, 106 (2002).

[17] J. Rudnick and R. Bruinsma, Phys. Rev. E 65, 030902(R) (2002).

[18] For a relevant application in the nonlinear optics of waveguide arrays, see, e.g., D. N. Christodoulides and R. I. Joseph, Opt. Lett. 13, 794 (1988). For a different application (BoseEinstein condensates in an optical lattice) with a similar spirit (i.e., geometrically dependent interaction with a coefficient dependent on the proximity between the nodes) see, e.g., A. Smerzi and A. Trombettoni, Chaos 13, 766 (2003). For a more mathematical treatment of this topic, see also G. L. Alfimov et al., Phys. Rev. E 66, 046608 (2002).

[19] T. Dauxois, Phys. Lett. A 159, 390 (1991).

[20] J. Cuevas et al., J. Phys. A 35, 10519 (2002).

[21] O. M. Braun and Yu. S. Kivshar, Phys. Rev. A 43, 1060 (1991).

[22] A. A. Maradudin, E. W. Montroll, and G. H. Weiss, Theory of Lattice Dynamics in the Harmonic Approximation (Academic Press, New York, 1963).

[23] J. L. Marín and S. Aubry, Nonlinearity 9, 1501 (1996).

[24] A. A. Sukhorukov, Phys. Rev. E 63, 016615 (2001).

[25] J. L. Marín and S. Aubry, Physica D 119, 163 (1996).

[26] J. Cuevas, Ph.D. thesis, University of Sevilla, Spain, 2003 (in Spanish).

[27] T. Cretegny, Ph.D. thesis, École normale supérieure de Lyon, France, 1998 (in French); see also M. Johansson and Yu. S. Kivshar, Phys. Rev. Lett. 82, 85 (1999).

[28] D. Chen, S. Aubry, and G. P. Tsironis, Phys. Rev. Lett. 77, 4776 (1996).

[29] J. Cuevas et al., Theor. Math. Phys. 137, 1406 (2003).

[30] P. G. Kevrekidis, Y. S. Kivshar, and A. S. Kovalev, Phys. Rev. E 67, 046604 (2003). 